\title{
Mapping momentum-dependent electron-phonon coupling and nonequilibrium phonon dynamics with ultrafast electron diffuse scattering
}

\author{
Mark J. Stern, ${ }^{1}$ Laurent P. René de Cotret, ${ }^{1}$ Martin R. Otto, ${ }^{1}$ Robert P. Chatelain, ${ }^{1}$ Jean-Philippe Boisvert, ${ }^{1}$ \\ Mark Sutton, ${ }^{1}$ and Bradley J. Siwick ${ }^{1,2, *}$ \\ ${ }^{1}$ Department of Physics, Center for the Physics of Materials, McGill University, 3600 University Street, Montreal, Quebec, Canada H3A 2T8 \\ ${ }^{2}$ Department of Chemistry, McGill University, 801 Sherbrooke Street W, Montreal, Quebec, Canada H3A OB8
}

(Received 5 October 2017; revised manuscript received 12 February 2018; published 12 April 2018)

\begin{abstract}
Despite their fundamental role in determining material properties, detailed momentum-dependent information on the strength of electron-phonon and phonon-phonon coupling (EPC and PPC, respectively) across the entire Brillouin zone has remained elusive. Here we demonstrate that ultrafast electron diffuse scattering (UEDS) directly provides such information. By exploiting symmetry-based selection rules and time resolution, scattering from different phonon branches can be distinguished even without energy resolution. Using graphite as a model system, we show that UEDS patterns map the relative EPC and PPC strength through their profound sensitivity to photoinduced changes in phonon populations. We measure strong EPC to the $K$-point TO phonon of $A_{1}^{\prime}$ symmetry $\left(K-A_{1}^{\prime}\right)$ and along the entire TO branch between $\Gamma-K$, not only to the $\Gamma-E_{2 g}$ phonon. We also determine that the subsequent phonon relaxation of these strongly coupled optical phonons involve three stages: decay via several identifiable channels to TA and LA phonons $(1-2 \mathrm{ps})$, intraband thermalization of the non-equilibrium TA/LA phonon populations (30-40 ps) and interband relaxation of the TA/LA modes (115 ps). Combining UEDS with ultrafast angle-resolved photoelectron spectroscopy will yield a complete picture of the dynamics within and between electron and phonon subsystems, helping to unravel complex phases in which the intertwined nature of these systems has a strong influence on emergent properties.
\end{abstract}

DOI: 10.1103/PhysRevB.97.165416

\section{INTRODUCTION}

The nature of the couplings within and between lattice and charge degrees of freedom is a central concern of condensedmatter and materials physics [1]. Electron-phonon interactions play a dominant role in the electronic transport properties of metals [2], and are the underlying cause of conventional superconductivity [3], Peierls/Jahn-Teller instabilities [4,5], and, in some cases, thermoelectrics [6]. Furthermore, they are central to our understanding of the properties of many quasiparticles including polarons [7] and phonon-polaritons [8]. Highly anisotropic (momentum-dependent) electron-phonon coupling (EPC) has been identified as a key feature of superconductivity in $\mathrm{MgB}_{2}$ [9]. It is also intertwined with electron correlations in the iron-based superconductor FeSe [10] and has been shown to contribute to the selection of the electronic ordering vector in some charge density wave materials including $\mathrm{ErTe}_{3}$ [11] and $\mathrm{NbSe}_{2}$ [12]. On the other hand, phonon-phonon coupling (PPC) dictates the thermalization properties of carrier/quasiparticle excitation energy.

The subtle details of charge-lattice interactions can have an enormous impact on technologically relevant material properties, but these interactions have so far resisted a comprehensive experimental investigation. Conventional angleresolved photoemission spectroscopy (ARPES) [13], inelastic $\mathrm{x}$-ray/neutron scattering [1], and Raman spectroscopy [14] provide indirect information on the EPC strength through the shifting and broadening of spectral features only over a

*bradley.siwick@mcgill.ca limited part of the Brillouin zone (BZ) [1]. Detailed studies of phonon-phonon interactions and decay in materials has typically been the province of theory [15] or molecular dynamics simulations due to a lack of techniques capable of probing these interactions in any substantial detail. Time-domain approaches have recently opened new windows on the nature of EPC and PPC in materials. Time-resolved Raman spectroscopy has been used to directly measure the rate of energy exchange between photogenerated carriers and zone-center optical phonons and the subsequent relaxation of those specific nonequilibrium phonons $[14,16,17]$. Time-resolved inelastic $x$-ray scattering at synchrotron and $\mathrm{x}$-ray free-electron laser facilities has provided a view of nonequilibrium distributions of off-zone-center phonons in InP and InSb [18] and the phonon band structure in Ge $[12,19]$ through incoherent and coherent time-resolved diffuse scattering signals, respectively.

Here we demonstrate that ultrafast electron diffuse scattering (UEDS) using radio-frequency compressed electron pulses provides a general, laboratory-scale, time-resolved analog of diffuse $x$-ray scattering [20]. This new method [21-23] is capable of directly determining both the relative momentumdependent interaction strength between photogenerated carriers and phonons and the subsequent phonon-phonon interactions governing the relaxation and thermalization of the excitation energy across the entire BZ with $\sim 100 \mathrm{fs}$ time resolution [24]. In pump-probe geometry [25-27] these experiments map the transient changes to diffuse (inelastic) electron scattering patterns in momentum space, which are themselves determined by the evolution of the nonequilibrium phonon distributions that follow electronic excitation. We show that this technique is particularly well suited as a probe of 
(a)

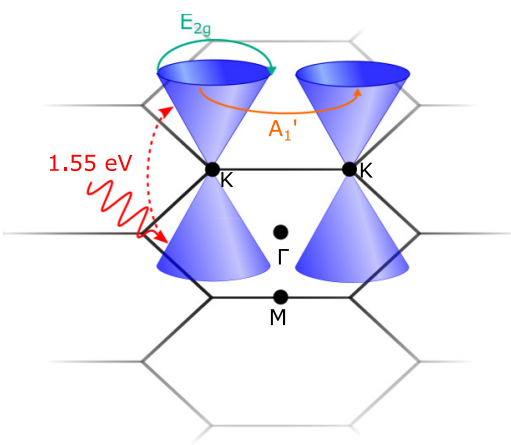

(b)

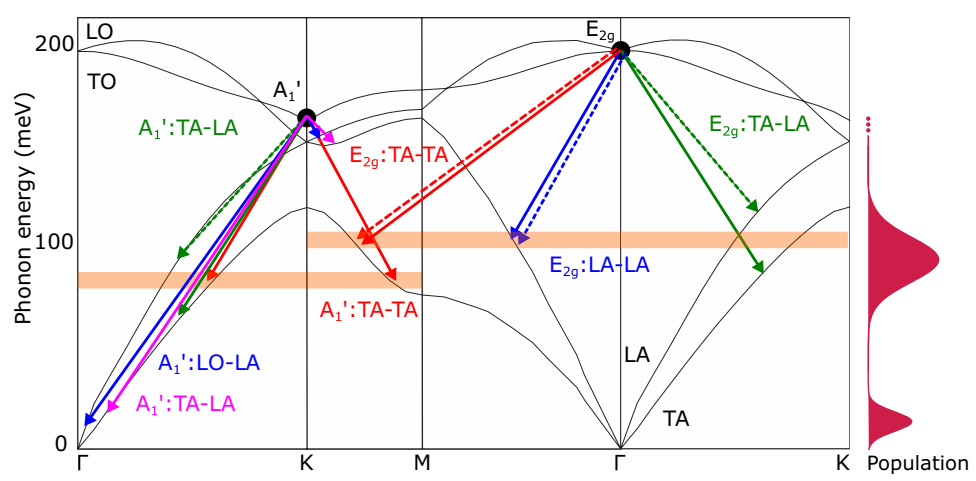

FIG. 1. Electron-phonon coupling and phonon-phonon coupling in graphite. (a) Simplified schematic of the electronic band structure of graphite illustrating the influence of the Dirac cones on the electron-phonon scattering process. In these experiments a pump laser pulse drives vertical electronic transitions $\left(\pi-\pi^{\star}\right)$. The resulting hot electrons (see text) may inelastically scatter with the $\Gamma-E_{2 g}$ phonon across a Dirac cone and with the $K-A_{1}^{\prime}$ phonon between Dirac cones, conserving energy and momentum. (b) Phonon dispersion of graphite with the strongly coupled optical modes indicated. The orange bars indicate half the energy of the $K-A_{1}^{\prime}$ and $\Gamma-E_{2 g}$ modes. The dominant energy and momentum-conserving decay pathways are indicated with colored arrows. For example, the green arrows labeled $A_{1}^{\prime}$ :TA-LA on the left represent the decay from the strongly coupled $A_{1}^{\prime}$ mode at $K$ to both TA and LA modes at the midpoint of the $\Gamma-K$ line. Dotted arrows should be thought of as going in the opposite momentum direction. The side bar (red) provides a schematic of the nonequilibrium LA/TA phonon distribution produced through the decay of $\Gamma-E_{2 g}$ and $K-A_{1}^{\prime}$ phonons as determined by the UEDS data described in the text.

two-dimensional (2D) materials using thin graphite as a model system. Specifically, optical excitation at $800 \mathrm{~nm}$ with $35 \mathrm{fs}$ laser pulses drives vertical electronic transitions of a $\pi-\pi^{\star}$ character on the well-known Dirac cones [28] of single-crystal graphite samples [Fig. 1(a)]. This excitation impulsively photodopes the material with a nonequilibrium electron-hole plasma with carrier density controllable by excitation fluence. Time-resolved ARPES (TR-ARPES) experiments have found that the first stage of relaxation is for the nonequilibrium distribution of carriers to thermalize internally through carriercarrier scattering, forming a Fermi-Dirac distribution with well-defined electron temperature within $\sim 50$ fs $[17,29,30]$. In this work we use UEDS patterns to determine how the energy stored in the hot electron system couples to the phonons and how the phonon system subsequently thermalizes, comparing the results - where possible — with earlier investigations using time-resolved Raman spectroscopy [14,17], pump-probe spectroscopy [31,32], and theory [15].

\section{RESULTS AND DISCUSSION}

The photoinduced changes to the ultrafast electron scattering pattern, $\Delta I(\boldsymbol{q}, \tau)=\frac{I(\boldsymbol{q}, \tau)-I(\boldsymbol{q},-\infty)}{I(\boldsymbol{q},-\infty)}$, are shown for several points in time following photoexcitation of a 50-nm-thick graphite film $\left(12 \mathrm{~mJ} / \mathrm{cm}^{2}, 800 \mathrm{~nm}\right)$ in Fig. 2. The evolution of these patterns from 0.5 to $100 \mathrm{ps}$ is striking and encodes detailed information on changes in the phonon system. The diffuse scattering intensity at scattering vector, $\boldsymbol{q}$, is determined by the population dynamics of phonon modes with momentum $\boldsymbol{k}$ according to [20]

$$
I(\boldsymbol{q}) \propto \sum_{j=1} \frac{\left(n_{j, \boldsymbol{k}}+\frac{1}{2}\right)}{\omega_{j, \boldsymbol{k}}} \underbrace{\left|\sum_{s} \frac{f_{s}}{\sqrt{\mu_{s}}} \exp \left(-M_{s}\right)\left(\boldsymbol{q} \cdot \hat{\mathbf{e}}_{j, s, \boldsymbol{q}}\right)\right|^{2}}_{\left|F_{j}(\boldsymbol{q})\right|^{2}} .
$$

The first sum is taken over all 12 graphite phonon branches, $j$, and the second is taken over the four atoms in the unit cell, $s . f_{s}$ is the atomic scattering factor, $\mu_{s}$ is the atomic mass, and $M_{s}$ is the Debye-Waller factor. Most important are $\omega_{j, \boldsymbol{k}}$ and $\hat{\mathbf{e}}_{j, \boldsymbol{k}}$, the momentum-dependent phonon frequency and polarization for branch $j$. Finally, $n_{j, k}$ is the population of the phonon mode with frequency $\omega_{j, k}$, which can evolve rapidly following photoexcitation. As shown in Eq. (1), scattered intensity is proportional to the instantaneous occupancy of the $\omega_{j, k}$ modes, multiplied by the norm of the one-phonon structure factor, $F_{j}(\boldsymbol{q})$. In general, the occupancy of a specific branch, $n_{j, k}$, is not directly available from the inelastic scattering signal at $\boldsymbol{q}$, since all modes $j$ contribute to $I(\boldsymbol{q})$; diffuse scattering is momentum resolved but energy integrated. With a more sophisticated analysis, however, the complete phonon band structure can be determined. This has been done previously in silicon, by combining modeling and static thermal diffuse $\mathrm{x}$-ray scattering data [20]. Here we demonstrate a different approach. Symmetry-imposed inelastic scattering selection rules, which play an important role in neutron scattering [33,34], describe extinctions in $F_{j}(\boldsymbol{q})$ at particular points of the diffuse scattering pattern for a given phonon mode. If the symmetry group of the scattering vector, $\boldsymbol{q}$, is strict, several of the phonon modes can be inactive $\left[F_{j}(\boldsymbol{q})=0\right.$ ], reducing the number of phonon branches that can contribute at that point. Thus, graphite and other high-symmetry 2D materials are excellent candidates for UEDS. These selection rules have been used previously in inelastic x-ray diffraction experiments [35] to measure the energy dependence of individual branches in the phonon dispersion of graphite. Here, we use the same selection rules to separate the population dynamics of individual phonon branches without energy resolution.

An attractive feature of UEDS is that a discrete, strongly coupled mode yields a peak in the differential scattering maps at the BZ momentum position associated with the mode at short delay times; i.e., electronic excitation energy initially 

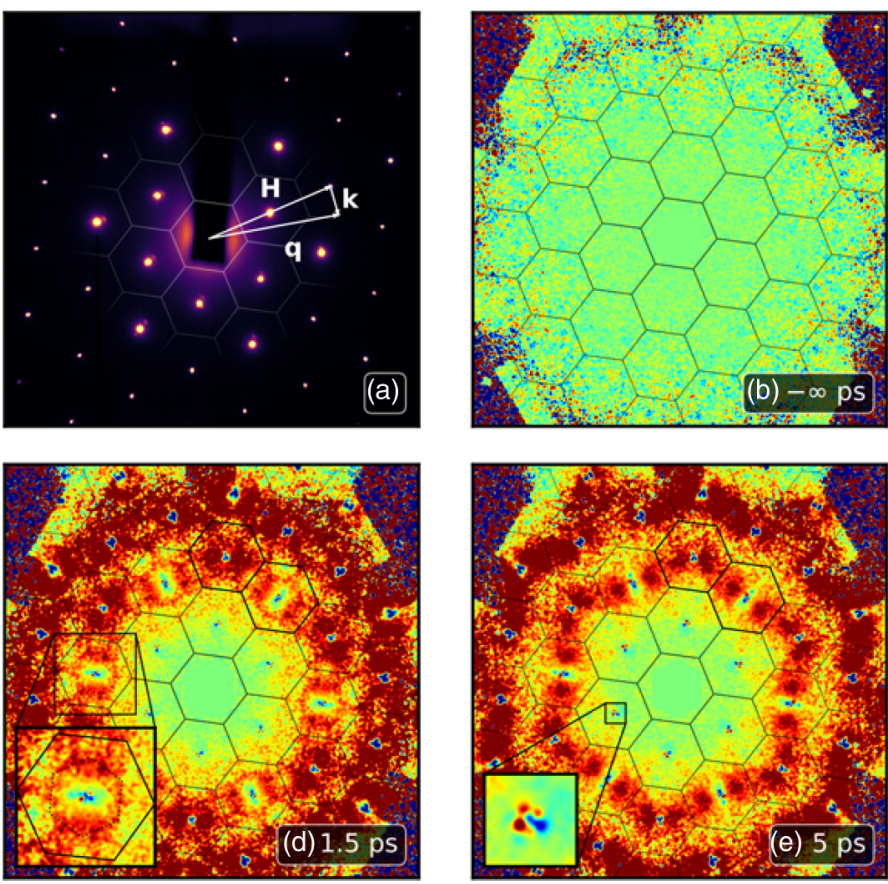
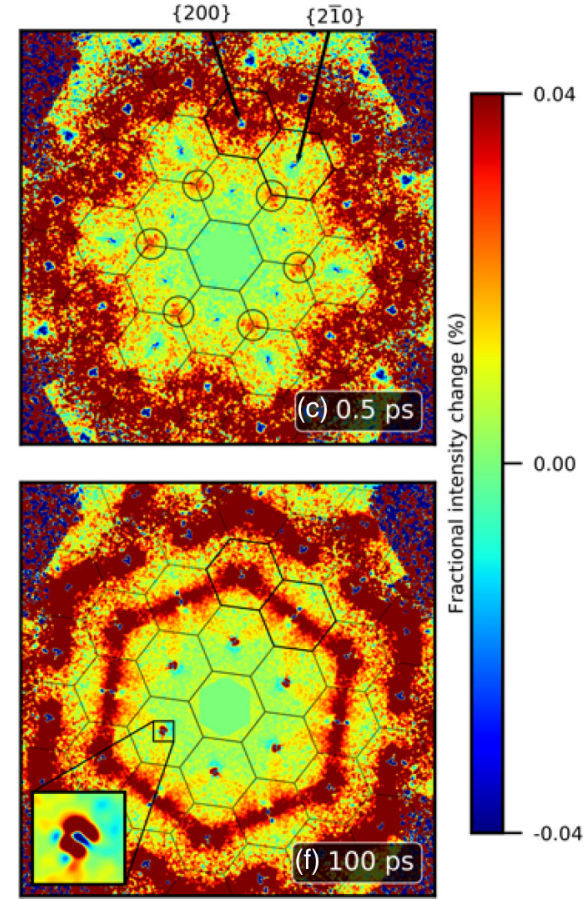

FIG. 2. Evolution of $\Delta I(\boldsymbol{q}, \tau)$ following photoexcitation of graphite (35 fs, $\left.800 \mathrm{~nm}, 12 \mathrm{~mJ} / \mathrm{cm}^{2}\right)$. The dramatic changes reflect the nonequilibrium phonon populations and their time dependence. (a) Raw diffraction pattern of graphite along the [001] zone axis showing sixfold symmetry of the graphene planes. (b) Differential scattering flat field $\Delta I(\boldsymbol{q}, \tau)$ at a time before optical excitation indicating signal to noise. (c) $\Delta I(\boldsymbol{q}, 0.5 \mathrm{ps})$ provides a map of the relative strength of the $\boldsymbol{q}$-dependent EPC coupling through the increased occupancy of strongly coupled modes. Peaks in $\Delta I(\boldsymbol{q}, 0.5 \mathrm{ps})$ at the $K$ points surrounding $\{2 \overline{1} 0\}$ (circled) result from the increase in $K$ - $A_{1}^{\prime}$ population and outline the hexagonal BZ. Scattering from the $\Gamma-E_{2 g}$ TO phonon is forbidden around $\{2 \overline{1} 0\}$, but strong coupling to the entire TO branch is evident in the vicinity of $\{200\}$ as ridges of intensity radiating from $\Gamma$ (the Bragg peak) to $K$ points. (d)-(f) Nonequilibrium phonon dynamics: relaxation of the transient population of strongly coupled optical modes. (d) At 1.5 ps the peaks evident at $K$ points in panel (c) have disappeared and diffuse intensity now appears halfway between $\{2 \overline{1} 0\}$ and the BZ edge (inset), but is still absent in the $M$ and $\Gamma_{\{2 \overline{1} 0\}}$ regions. (e) By 5 ps, the character of $\Delta I(\boldsymbol{q}, \tau)$ has changed dramatically to bands of intensity in the $\Gamma_{\{2 \overline{1} 0\}}-M-\Gamma_{\{200\}}$ direction approximately orthogonal to $\boldsymbol{q}$, with troughs near $\Gamma_{\{2 \overline{1} 0\}}$ remaining. (f) At $100 \mathrm{ps}$ the $\Gamma_{\{2 \overline{1} 0\}}-M-\Gamma_{\{200\}}$ bands have become sharper and the troughs near $\Gamma_{\{210\}}$ have filled in. Strong halos of diffuse intensity are present around the $\{100\}$ and $\{110\}$ families of peaks are evident (inset). These halos are weak, but present at 5 ps [inset, panel (e)].

flows preferentially to modes with strong EPC and these are the first to show an increase in diffuse scattering [Fig. 2(c)]. Here, we observe diffuse scattering peaks $\left(\mathrm{FWHM}=0.12 \AA^{-1}\right)$ at the $K$ points along the reflection axes, near the $\{2 \overline{1} 0\}$ family of peaks (where scattering from the $K-A_{1}^{\prime}$ mode is allowed) and along starlike ridges joining $\Gamma_{\{200\}}-K$ (where TO branch scattering is allowed). The phonon dispersion relation of graphite [Fig. 1(b)] shows strong softening of the LO branch near $\Gamma$ and the TO branch near $K$ due to Kohn anomalies [36-38]. Earlier work suggested these strongly coupled modes as the initial reservoir into which the electronic excitation energy flows [32] and our results confirm that hypothesis. Time-resolved Raman has previously been employed to follow the the occupancy of the zone center $\Gamma-E_{2 g}$ mode showing that it is indeed strongly coupled [14,17]. Evidence for strong coupling to the off-zone-center $K-A_{1}^{\prime}$ mode, however, has previously only been indirect. The peaks in Fig. 2(c) represent a direct observation of this effect. In addition, Fig. 2(c) indicates that coupling is strong for the entire TO branch between $\Gamma-K$, not only for the $\Gamma-E_{2 g}$ mode.

The character of the differential diffuse scattering pattern changes dramatically through Figs. 2(c)-2(f) as the nonequilibrium phonon distribution evolves, demonstrating the profound sensitivity to the details of the phonon occupancies. The complete time dependence of the diffuse intensity at selected BZ points is shown in Fig. 3. Scattering from the $K-A_{1}^{\prime}$ mode is forbidden by symmetry at the $K$ points immediately proximate to the reflection axes (indicated by green in the inset); only LO phonon scattering is observed at these points [35]. Thus, $\Delta I(\boldsymbol{q}, \tau)$ at this point shows a qualitatively distinct time dependence [Fig. 3(b), green] versus $K$ points along the reflection axes at which scattering from the $K-A_{1}^{\prime}$ mode is allowed [Fig. 3(b), red]. This includes a much slower initial rise; $730 \mathrm{fs}$ ( $K$-LO) compared to $280 \mathrm{fs}$ ( $K$-TO). Intensity near $\{200\}$ [Fig. 3(b), cyan] reports on the occupancy of the strongly coupled $\Gamma-E_{2 g}$ TO mode at early times, and exhibits a slower rise (430 fs) than the $K-A_{1}^{\prime}$ mode. For comparison, the $\Gamma-E_{2 g}$ phonon population determined using TR-Raman [14] is shown in gray. The $\Gamma-E_{2 g}$ phonon population dynamics determined by TR-Raman is nearly identical to that shown for the $K-A_{1}^{\prime}$ phonon population (red curve) in terms of both rise time and decay/recovery. The dynamics of both strongly coupled optical phonons (SCOPs) is very similar, showing a rapid increase in population due to strong EPC and then a subsequent depopulation via PPC and relaxation to lower frequencies. The slower rise time observed for the $\Gamma-E_{2 g}$ phonon by UEDS is 

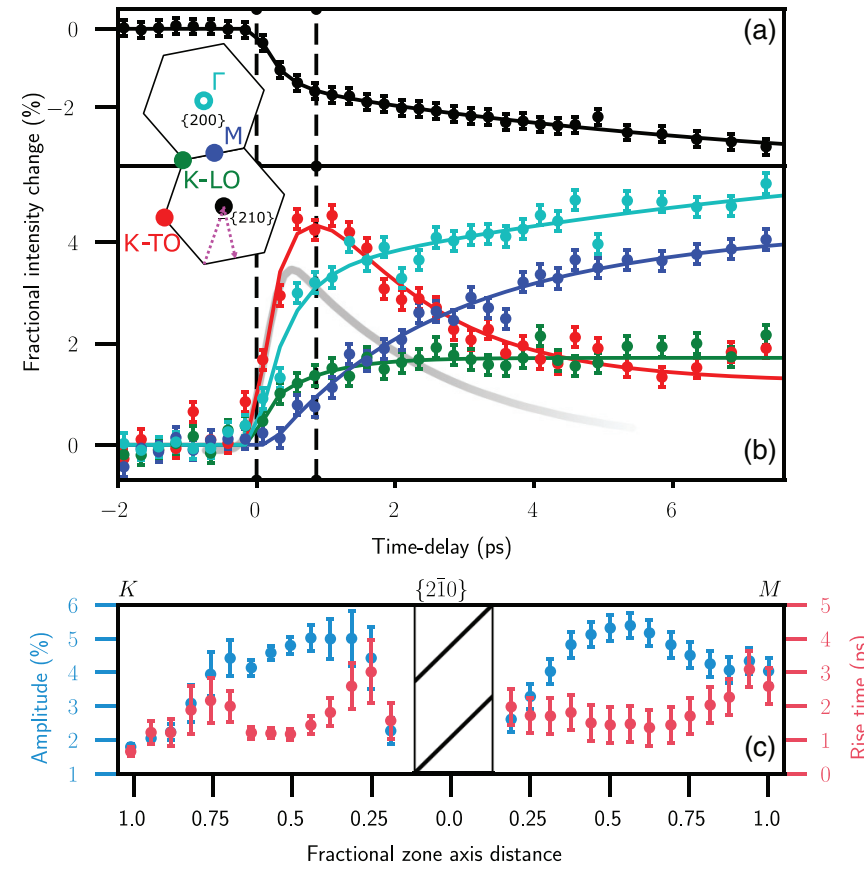

FIG. 3. Ultrafast electron diffuse scattering. (a) Intensity of the $\{2 \overline{1} 0\}$ Bragg peak showing profoundly nonexponential Debye-Waller dynamics [26]. (b) $\Delta I(\boldsymbol{q}, \tau)$ at select points in the BZ (inset). The rate of increase in the population (EPC) of the TO $K-A_{1}^{\prime}$ phonon (red, $280 \mathrm{fs}$ ) is faster than that for the TO $\Gamma-E_{2 g}$ (cyan, $430 \mathrm{fs}$ ) and matches the fast Bragg peak dynamics. The population $K$-LO phonons (green, $730 \mathrm{fs}$ ) rises much slower than both TO $K-A_{1}^{\prime}$ and TO $\Gamma-E_{2 g}$ phonons. The rise in diffuse intensity at the $M$ point (blue, 2.1 ps) is almost an order of magnitude slower than that associated with the TO $K-A_{1}^{\prime}$ phonon. The slow time-scale decay evident in the Bragg peak [26] and reported in earlier ARPES measurements [13] does not emerge from the dynamics of any single mode, but is a composite of the decay in population of the strongly coupled optical modes (e.g., red, $1.7 \mathrm{ps)}$ and the increase in population of all other modes. (c) Diffuse intensity dynamics for points along the $K-\Gamma$ line, and $\Gamma-M$ line (b) (inset, magenta). Rise time (blue) and amplitude (red) from single exponential fits to the early-time dynamics are shown. These dynamics are associated phonon-phonon decay (PPC) from the SCOPs to the acoustic modes. Error bars represent covariance in fit parameters. Points close to $\Gamma$ are not shown due to the interference of the Bragg peak Debye-Waller dynamics of (a).

likely due to the higher excitation conditions used $\left(12 \mathrm{~mJ} / \mathrm{cm}^{2}\right.$ compared to $0.2 \mathrm{~mJ} / \mathrm{cm}^{2}$ ) which is known to weaken the Kohn anomaly and EPC at the $\Gamma$ point $[14,31]$. Although the $\Gamma-E_{2 g}$ TO mode is also strongly coupled, the recovery of the red and gray curves is not seen in cyan. This difference is due to scattering from the low-frequency LA and TA phonon modes involved in the dominant decay channels of the $K-A_{1}^{\prime}$ TO phonon [Fig. 1(b)] that are observed at this momentum and discussed further below. These LA/TA modes are not seen in the TR-Raman study and do not overlap (i.e., are momentum separated) with the signals measured at the $K$ point.

We can estimate the effective temperature of the $K$-point TO mode, $T_{T O, K}$, using the measured increase in diffuse intensity and applying Bose-Einstein statistics to the mode population, $n_{j, k}=\operatorname{coth}\left(\hbar \omega_{j, k} / 2 k_{B} T_{j, k}\right)-\frac{1}{2}$. We determine that the effective temperature of the $K$-point TO mode is $\sim 1150 \mathrm{~K}$ by $1 \mathrm{ps}$; also by $10 \mathrm{ps}$ it has cooled back down to $\sim 500 \mathrm{~K}$. The strongly coupled TO and LO modes reach a preequilibrium with the laser-generated carriers in $<1 \mathrm{ps,} \mathrm{while} \mathrm{all} \mathrm{other}$ phonon modes remain at or near room temperature on this time scale.

The diffuse scattering patterns in Figs. 2(d)-2(f) reveal the decay channels for the population of strongly coupled optical phonons as they relax. The time-scale separation between the EPC into $K-A_{1}^{\prime}, \Gamma-E_{2 g}$, and the TO branch (200-400 fs) and the subsequent decay out of these modes (1-3 ps) means that the diffuse scattering pattern at $1.5 \mathrm{ps}$ effectively maps their momentum-dependent decay probability of the strongly coupled optical phonons in a manner analogous to the way in which the $0.5 \mathrm{ps}$ pattern indicates the relative EPC strength. The probability for the $\Gamma-E_{2 g}$ phonon to decay to two phonons of momentum $\boldsymbol{k}$ and $\boldsymbol{- k}$ was previously computed using density functional perturbation theory [15] for the distinct momentum and energy-conserving channels indicated by arrows in Fig. 1(b). The results of these calculations compare well with $\Delta I(\boldsymbol{q}, \tau=1.5 \mathrm{ps})$ in Fig. 2(d) (inset). The hexagonal distribution of diffuse intensity approximately halfway between $\Gamma$ and the BZ edges is associated with the TA-LA (green) and $E_{2 g}:$ LA-LA (red) decay channels. Peaks evident along the $\Gamma-K$ lines are also in agreement with the expected location of maximum decay rate. The time constants associated with each of these channels can be determined directly from the time dependence of $\Delta I(\boldsymbol{q}, \tau)$ at the associated $\boldsymbol{k}$ position in the BZ, shown for selected positions in Fig. 3(b). The variation in the rise time and amplitude of $\Delta I(\boldsymbol{q}, \tau)$ in the BZ surrounding the $\{2 \overline{1} 0\}$ peak is given along the $\Gamma-K$ and $\Gamma-M$ directions in Fig. 3(c). The exact path, chosen such that the TA mode structure factor is overwhelmingly dominant, is shown in Fig. 3(b) (inset). These dynamics should be understood as relating to the change in TA mode occupation that result from the decay of the SCOPs (PPC). Here, the prominent TA-LA (green) and $E_{2 g}^{\prime}:$ TA-TA (red) decay channels in the $\boldsymbol{k}=0.5 \mathrm{~K}$ and $\boldsymbol{k}=0.5 \mathrm{M}$ regions have the fastest time constants (1.0-1.5 ps) and largest amplitude.

The decay of the $K-A_{1}^{\prime}$ phonon population, evident in the relaxation of the $K$-TO intensity shown in Fig. 3(b) (red, $1.7 \mathrm{ps}$ ), is dominated by LO-LA (blue) and TA-LA (green/magenta) channels. Figure 4 shows the time dependence of the diffuse intensity adjacent $\left(\sim 0.4 \AA^{-1}\right)$ to the $\{200\}$ and $\{210\}$ peaks along the $\Gamma-K$ direction. Adjacent to $\{210\}$, scattering from $\Gamma-E_{2 g}$ is forbidden by symmetry; thus, the fastest time constant evident in the data, 1.5 ps [Fig. 4 (gray)], can only be assigned to the LO-LA (blue) and TA-LA (magenta) decay channels of the $K-A_{1}^{\prime}$ phonon. Adjacent to $\{200\}$, scattering from $\Gamma-E_{2 g}$ is allowed and the early-time dynamics appear approximately biexponential [Fig. 4 inset (cyan)]. The observed fast time-constant behavior is associated with the strong EPC to the $\Gamma-E_{2 g}$ mode, as previously described. The slower dynamics is assigned to a composite time scale resulting from a decrease in intensity due to the decay of $\Gamma-E_{2 g}$ phonons and an increase due to the LO-LA (blue) and TA-LA (magenta) decay channels of the $K-A_{1}^{\prime}$ phonon. It is clear that time resolution in UEDS can substitute for energy resolution when discriminating contributions from various phonon branches at a single momentum. 


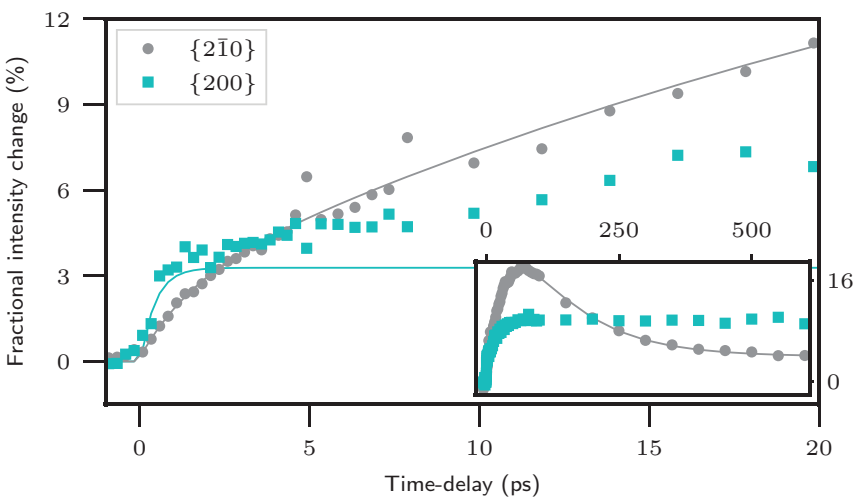

FIG. 4. Diffuse intensity near $\Gamma_{\{2 \overline{1} 0\}}$ (gray) and $\Gamma_{\{200\}}$ (cyan). Early-time diffuse intensity changes demonstrate how time resolution and selection rules can be used to separate the dynamics of phonon branches at the same momentum. The cyan curve displays a quasibiexponential character while the gray curve is a single exponential rise ( $1.5 \mathrm{ps}$ ). The fast cyan component (430 fs, solid line) has been assigned to EPC to the $\Gamma-E_{2 g}$ mode. This signal is absent from the gray curve due to symmetry-imposed selection rules. The $1.5 \mathrm{ps}$ rise in the gray curve (solid line) is associated with the low-frequency LA/TA populations generated through $K-A_{1}^{\prime}$ phonon decay. The longer time constant in the cyan curve is a composite time scale associated with the decay of the $\Gamma-E_{2 g}$ population and the increase in the low-frequency LA/TA populations. Inset: Diffuse intensity changes for the entire time range. The 34 ps rise in intensity in the gray curve is associated with the intraband thermalization of the nonequilibrium LA and TA populations generated through the decay of strongly coupled $\Gamma-E_{2 g}$ and $K-A_{1}^{\prime}$ phonons. The 115 ps decay most evident in the $\Gamma_{\{210\}}$ data is associated with interband equilibration of the phonon populations in the LA, TA, and ZA bands. Solid gray line shows triexponential fit to the 1.5 ps rise, $34 \mathrm{ps}$ rise, and 115 ps decay.

On longer time scales the dynamics evident in the phonon system remain rich. The early-time diffuse intensity bands oriented along the $\Gamma-K$ directions [Figs. 2(c) and 2(d)] evolve to become intensity bands oriented along the $\Gamma-M$ directions. The two principal factors in these dynamics are that the decay of the strongly coupled optical modes generates a profoundly nonequilibrium population of LA and TA phonons [Fig. 1(b)], and that these branches are considerably softer along the $\Gamma-M$ direction than the $\Gamma-K$ in graphite. The nonequilibrium populations of the LA and TA phonons thermalize within these bands by emission of lower-frequency LA and TA phonons. This relaxation channel is evident in the increase in the diffuse halos surrounding the Bragg peaks in Figs. 2(e) and 2(f) (inset), whose detailed time dependence is plotted in Fig. 4. This relaxation of the phonon distribution in the acoustic branches occurs on a time scale of $34 \mathrm{ps}$ and is a phonon analog of the carrier thermalization that has previously been observed in TR-ARPES experiments [17,29,30]. The lowest-frequency branch of the in-plane phonon band structure consists of acoustic modes with a polarization normal to the graphene planes (ZA modes), to which the current experiments are insensitive due to electron beam orientation and scattering selection rules [Eq. (1)]. Relaxation into the ZA branch and LA/TA interband relaxation is evident, however, due to the decay of the diffuse intensity near the $\{2 \overline{1} 0\}$ peak (Fig. 4 ) which occurs on the 115 ps time scale.
By $\sim 500$ ps, the phonon system has reached a quasisteady state, where intensity is roughly constant, but has not yet thermalized (Fig. 4 inset). The bands of diffuse intensity along the $\Gamma-M-\Gamma$ lines cannot be described with a single temperature, indicating that these modes are out of equilibrium. It should also be noted that the thermal transport of the laser deposited energy out of the probed volume occurs on the $10 \mu$ s time scale in this geometry, so almost all of the pump energy remains in the sample at $500 \mathrm{ps}$. At room temperature, diffuse scattering only occurs very close to the $\Gamma$ points due to the substantial hardness of modes at $k>0$. Therefore, even at the longest time scale in these experiments the in-plane phonon distribution is still profoundly nonequilibrium.

We have demonstrated that UEDS provides direct, momentum-resolved measurements of the relative strength of EPC and PPC in graphite through the technique's ability to follow phonon population dynamics with femtosecond time resolution. Particularly notable is the unprecedented picture of phonon decay kinetics that the UEDS patterns provide, only a summary of which has been given here. UEDS is also profoundly complementary to ultrafast ARPES. Together these methods can provide a complete picture of the dynamics within and between electron and phonon subsystems, and help unravel the physics of complex phases where the intertwined nature the electron-lattice systems determine material properties.

\section{MATERIALS AND METHODS}

\section{A. Ultrafast electron diffraction}

The ultrafast electron diffractometer used in this work is described in detail elsewhere [24,25]. Briefly, the instrument consists of an amplified ultrafast laser system (Spectra-Physics XP) and a home-built $100 \mathrm{keV}$ electron source. The output from the amplified laser is split into two beams. The first beam is frequency tripled to produce ultraviolet light, which is made incident on a copper photocathode in the electron source. The photogenerated electron pulses produced $\left(\sim 10^{6} /\right.$ pulse $)$ serve as the probe after compression with a $3 \mathrm{GHz}$ synchronized microwave field in a $\mathrm{TM}_{010}$ cavity. A Gatan Ultrascan 895 camera detects the UEDS patterns that are produced. The second beam passes through an optical delay line (translation stage) and is used to photoexcite the sample (pump) at a controllable time $\Delta \tau$ relative to the arrival of the electron probe pulse. The data presented in Fig. 2 represents the accumulated signal from approximately 500000 pump-probe cycles (or 15 min per time point).

\section{B. Data analysis}

Relative change patterns $\Delta I(\boldsymbol{q}, \tau)$ are calculated as follows:

$$
\Delta I(\boldsymbol{q}, \tau)=\frac{I(\boldsymbol{q}, \tau)-\frac{1}{n} \sum_{t<0} I(\boldsymbol{q}, t)}{\frac{1}{n} \sum_{t<0} I(\boldsymbol{q}, t)},
$$

where $I(\boldsymbol{q}, \tau)$ is the diffraction measurement at time delay $\tau$, and $n$ is the number of measurements for which $t<0$. The relative change patterns presented in Fig. 2 present the raw data after sixfold rotational averaging and are smoothed with a 4 pixel ( $0.03 \AA$ ) Gaussian kernel, for visualization purposes only. Smoothing was not used in the extraction of the time series on Figs. 3 and 4. 
All data processing and analysis code is based on the opensource scientific Python stack [39,40] and is available upon reasonable request.

\section{Sample preparation}

Single-crystal flakes of natural graphite (Naturally Graphite $($ ) 10-50 nm thick were prepared using an exfoliation procedure. Thick flakes are first embedded in Crystalbond ${ }^{\mathrm{TM}}$ glue on a $3 \mathrm{~mm}$ transmission electrom microscopy copper grid (200 lines/in.). These thick, fixed flakes are then exfoliated using adhesive tape until transparent under a microscope. Once suitably transparent samples are obtained, the fixing agent $\left(\right.$ Crystalbond $^{\mathrm{TM}}$ ) is washed away with acetone. Sample thickness has been measured directly using atomic force microscopy characterization.

\section{ACKNOWLEDGMENTS}

This work was supported by the Natural Sciences and Engineering Research Council of Canada (NSERC), the Fonds de Recherche du Québec-Nature et Technologies (FRQNT), the Canada Foundation for Innovation (CFI), and Canada Research Chairs (CRC) program.

M.J.S. and L.P.R. de C. contributed equally to this work.
[1] T. P. Devereaux, A. M. Shvaika, K. Wu, K. Wohlfeld, C. J. Jia, Y. Wang, B. Moritz, L. Chaix, W.-S. Lee, Z.-X. Shen et al., Phys. Rev. X 6, 041019 (2016)

[2] G. Grimvall, The Electron-Phonon Interaction in Metals (NorthHolland, Amsterdam, 1981).

[3] J. Bardeen, L. N. Cooper, and J. R. Schrieffer, Phys. Rev. 108, 1175 (1957)

[4] R. Peierls, Ann. Phys. (NY) 396, 121 (1930).

[5] G. A. Gehring and K. A. Gehring, Rep. Prog. Phys. 38, 1 (1975).

[6] H. Wang, Y. Pei, A. D. LaLonde, and G. J. Snyder, Proc. Natl. Acad. Sci. USA 109, 9705 (2012).

[7] A. S. Alexandrov and J. T. Devreese, Advances in Polaron Physics (Springer, New York, 2010).

[8] S. Dai, Z. Fei, Q. Ma, A. S. Rodin, M. Wagner, A. S. McLeod, M. K. Liu, W. Gannett, W. Regan, K. Watanabe et al., Science 343, 1125 (2014).

[9] T. Yildirim, O. Gülseren, J. W. Lynn, C. M. Brown, T. J. Udovic, Q. Huang, N. Rogado, K. A. Regan, M. A. Hayward, J. S. Slusky et al., Phys. Rev. Lett. 87, 037001 (2001).

[10] S. Gerber, S.-L. Yang, D. Zhu, H. Soifer, J. A. Sobota, S. Rebec, J. J. Lee, T. Jia, B. Moritz, C. Jia et al., Science 357, 71 (2017).

[11] H.-M. Eiter, M. Lavagnini, R. Hackl, E. A. Nowadnick, A. F. Kemper, T. P. Devereaux, J.-H. Chu, J. G. Analytis, I. R. Fisher, and L. Degiorgi, Proc. Natl. Acad. Sci. USA 110, 64 (2013).

[12] X. Zhu, Y. Cao, J. Zhang, E. W. Plummer, and J. Guo, Proc. Natl. Acad. Sci. USA 112, 2367 (2015).

[13] S.-I. Tanaka, M. Matsunami, and S.-I. Kimura, Sci. Rep. 3, 3031 (2013).

[14] H. Yan, D. Song, K. F. Mak, I. Chatzakis, J. Maultzsch, and T. F. Heinz, Phys. Rev. B 80, 121403 (2009).

[15] N. Bonini, M. Lazzeri, N. Marzari, and F. Mauri, Phys. Rev. Lett. 99, 176802 (2007).

[16] K. T. Tsen and D. K. Ferry, J. Phys.: Condens. Matter 21, 174202 (2009).

[17] J.-A. Yang, S. Parham, D. Dessau, and D. Reznik, Sci. Rep. 7, 40876 (2017).

[18] M. Trigo, J. Chen, V. H. Vishwanath, Y. M. Sheu, T. Graber, R. Henning, and D. A. Reis, Phys. Rev. B 82, 235205 (2010).

[19] M. Trigo, M. Fuchs, J. Chen, M. P. Jiang, M. Cammarata, S. Fahy, D. M. Fritz, K. Gaffney, S. Ghimire, A. Higginbotham et al., Nat. Phys. 9, 790 (2013).

[20] M. Holt, Z. Wu, H. Hong, P. Zschack, P. Jemian, J. Tischler, H. Chen, and T.-C. Chiang, Phys. Rev. Lett. 83, 3317 (1999).

[21] T. Chase, M. Trigo, A. H. Reid, R. Li, T. Vecchione, X. Shen,
S. Weathersby, R. Coffee, N. Hartmann, D. A. Reis et al., Appl. Phys. Lett. 108, 041909 (2016).

[22] L. Waldecker, R. Bertoni, H. Hübener, T. Brumme, T. Vasileiadis, D. Zahn, A. Rubio, and R. Ernstorfer, Phys. Rev. Lett. 119, 036803 (2017).

[23] M. Harb, H. Enquist, A. Jurgilaitis, F. T. Tuyakova, A. N. Obraztsov, and J. Larsson, Phys. Rev. B 93, 104104 (2016).

[24] M. R. Otto, L. P. R. de Cotret, M. J. Stern, and B. J. Siwick, Struct. Dyn. 4, 051101 (2017).

[25] R. P. Chatelain, V. R. Morrison, C. Godbout, and B. J. Siwick, Appl. Phys. Lett. 101, 081901 (2012).

[26] R. P. Chatelain, V. R. Morrison, B. L. M. Klarenaar, and B. J. Siwick, Phys. Rev. Lett. 113, 235502 (2014).

[27] V. R. Morrison, R. P. Chatelain, K. L. Tiwari, A. Hendaoui, A. Bruhacs, M. Chaker, and B. J. Siwick, Science 346, 445 (2014).

[28] A. H. Castro Neto, F. Guinea, N. M. R. Peres, K. S. Novoselov, and A. K. Geim, Rev. Mod. Phys. 81, 109 (2009).

[29] I. Gierz, F. Calegari, S. Aeschlimann, M. Chávez Cervantes, C. Cacho, R. T. Chapman, E. Springate, S. Link, U. Starke, C. R. Ast et al., Phys. Rev. Lett. 115, 086803 (2015).

[30] A. Stange, C. Sohrt, L. X. Yang, G. Rohde, K. Janssen, P. Hein, L.-P. Oloff, K. Hanff, K. Rossnagel, and M. Bauer, Phys. Rev. B 92, 184303 (2015).

[31] K. Ishioka, M. Hase, M. Kitajima, L. Wirtz, A. Rubio, and H. Petek, Phys. Rev. B 77, 121402 (2008).

[32] T. Kampfrath, L. Perfetti, F. Schapper, C. Frischkorn, and M. Wolf, Phys. Rev. Lett. 95, 187403 (2005).

[33] J. M. Perez-Mato, M. Aroyo, J. Hlinka, M. Quilichini, and R. Currat, Phys. Rev. Lett. 81, 2462 (1998).

[34] A. K. Kirov, M. I. Aroyo, and J. M. Perez-mato, J. Appl. Crystallogr. 36, 1085 (2003).

[35] J. Maultzsch, S. Reich, C. Thomsen, H. Requardt, and P. Ordejón, Phys. Rev. Lett. 92, 075501 (2004).

[36] S. Pisana, M. Lazzeri, C. Casiraghi, K. S. Novoselov, A. K. Geim, A. C. Ferrari, and F. Mauri, Nat. Mater. 6, 198 (2007).

[37] S. Piscanec, M. Lazzeri, F. Mauri, A. C. Ferrari, and J. Robertson, Phys. Rev. Lett. 93, 185503 (2004).

[38] A. Politano, F. de Juan, G. Chiarello, and H. A. Fertig, Phys. Rev. Lett. 115, 075504 (2015).

[39] S. van der Walt, S. C. Colbert, and G. Varoquaux, Comput. Sci. Eng. 13, 22 (2011).

[40] S. van der Walt, J. L. Schönberger, J. Nunez-Iglesias, F. Boulogne, J. D. Warner, N. Yager, E. Gouillart, T. Yu, and the scikit-image contributors, PeerJ 2, e453 (2014). 